\title{
ON COMPACTNESS IN FUNCTION SPACES
}

\author{
GIOVANNI VIDOSSICH
}

\begin{abstract}
There are shown some implications from pseudocompactness to compactness or sequential compactness. The latter, sequential compactness, is obtained via metrization.
\end{abstract}

In [4] there are a number of criteria for function spaces and topological vector spaces showing that countable compactness implies compactness or sequential compactness. Since pseudocompactness weakens countable compactness, it seems natural to ask whether pseudocompactness leads to the same implications. For example, in [8] it is proved that in a complete locally convex space a weakly pseudocompact subset is relatively weakly compact. The aim of this note is to give some positive answer to the above question. We shall only deal with the topology of pointwise convergence, since one can easily deduce from our results similar ones for the topology of uniform convergence on compacta or more general subsets of the domain.

Notations. We shall denote by

$C_{p}(X, Y)$ the topological space of all continuous maps $X \rightarrow Y$ with the topology of pointwise convergence,

$C_{p}(X)$ the space $C_{p}(X, R)$.

\section{Compactness.}

1.1 THEOREM. Let $X$ be a topological space with the following property:

(*) $f: X \rightarrow \boldsymbol{R}$ is continuous iff. for each countable subset $C$ of $X,\left.f\right|_{C}$ is continuous.

Let $Y$ be a uniformizable space and $H \subseteq C(X, Y)$. Then $H$ is relatively compact in $C_{p}(X, Y)$ iff $H$ is relatively pseudocompact and, for every $x \in X, \operatorname{cl}(H(x))$ is complete for the fine uniformity of $Y$.

Recall that a topological space $X$ is said to be pseudocompact iff every continuous map $X \rightarrow R$ is bounded, while $A \subseteq X$ is said to be relatively pseudocompact iff $\bar{A}$ is pseudocompact. The fine uniformity is the finest compatible uniformity.

Received by the editors December 4, 1970.

AMS 1970 subject classifications. Primary 54C35, 54D30; Secondary 54E35, 46A99. Key words and phrases. Function spaces, compactness, pseudocompactness, countable compactness, sequential compactness, metrization, product of separable spaces.

c) American Mathematical Society 1972 
Examples of spaces $X$ with property (*) are: first countable spaces and (by [7] and a remark at [3, pp. 789-790]) products of separable metric spaces. Moreover, any separable (=there is a countable dense subset) space, hence any sum of separable spaces, has property (*), as one can easily see by a well-known theorem on extension from dense subsets of continuous mappings into regular spaces. Therefore we have the following extension of $[7, \mathrm{II}]$ to noncomplete spaces $X$ 's:

1.2 Corollary. Let $X$ be any locally convex space. If $X^{\prime}$ is weakly* separable, then for the weak topology of $X$ relative compactness and relative pseudocompactness are the same.

Finally, the above theorem generalizes [4, Theorem 2] to noncompact domains, since the closure of a relatively countably compact subset is pseudocompact. Note that our proof is somewhat simpler than the proof of [4, Theorem 2].

Proof of 1.1. The necessity being clear, we prove only the sufficiency.

Case 1. $Y$ is Hausdorff (hence completely regular). Let $\bar{H}$ be the closure of $H$ in $C_{p}(X, Y)$. Since $\bar{H}(x)=\{f(x) \mid f \in \bar{H}\}$ is a continuous image of $\bar{H}$, $\vec{H}(x)$ is pseudocompact. Since $\vec{H}(x)$ is dense in $\mathrm{cl}(H(x)), \operatorname{cl}(H(x))$ is pseudocompact. Consequently $\mathrm{cl}(H(x))$ is compact by the precompactness of compatible uniformities on a pseudocompact space. Therefore $\prod_{x \in X} \mathrm{cl}(H(x))$ is compact by Tychonoff theorem. Then we have only to show that each $f$ in the closure $H^{*}$ of $H$ in $\prod_{x \in X} \operatorname{cl}(H(x))$ is a continuous map $X \rightarrow Y$. Since $Y$ is completely regular, $Y$ is embeddable into a product $\prod_{\alpha \in A} R$ of copies of the reals. Then: $f: X \rightarrow Y$ is continuous iff $p_{\alpha} \circ f, p_{\alpha}$ being the canonical projection $\prod_{\alpha} \boldsymbol{R} \rightarrow \boldsymbol{R}$, is continuous for every $\alpha \in A$. Choose $f_{0} \in H^{*}$, and $\alpha \in A$. The subspace $H_{\alpha}=\left\{p_{\alpha} \circ f \mid f \in \bar{H}\right\}$ of $C_{p}(X)$ is pseudocompact since it is a continuous image of $\bar{H}$. By (*), $p_{\alpha} \circ f_{0}$ is continuous iff, for each countable $C \subseteq X,\left.p_{\alpha} \circ f\right|_{C}$ is continuous. Choose then a countable $C \subseteq X$. Let

$$
r: \prod_{x \in X} \boldsymbol{R} \rightarrow \prod_{x \in C} \boldsymbol{R}
$$

be the projection $f \leadsto \rightarrow f_{\mid C}$. Since $r$ is continuous, $r\left(H_{\alpha}\right)$ is a pseudocompact subspace of $C_{p}(C)$. Since $C$ is countable, $C_{p}(C)$ is metrizable. Since a metrizable pseudocompact space is compact, $r\left(H_{\alpha}\right)$ is compact. Then $r\left(H_{\alpha}\right)$ is a closed subset of $\prod_{x \in C} R$. Therefore $r\left(p_{\alpha} \circ f_{0}\right) \in r\left(H_{\alpha}\right)$, so that there is $g \in H_{\alpha}$ such that $g(x)=p_{\alpha}\left(f_{0}(x)\right)$ for all $x \in C$. Thus $p_{\alpha} \circ f_{0} \mid c$ is continuous.

Case 2. $Y$ is not Hausdorff. Let $\sim$ be the equivalence relation

$$
\left.y^{\prime} \sim y^{\prime \prime} \Leftrightarrow \overline{\left\{y^{\prime}\right\}}=\overline{\left\{y^{\prime \prime}\right.}\right\}
$$

on $Y$. Let $Y \mid \sim$ be the quotient space and $q: Y \rightarrow Y \mid \sim$ the quotient map. 
Since $H_{0}=\{g \circ f \mid f \in H\}$ is a continuous image of $H, H_{0}$ is a relatively pseudocompact subspace of $C_{p}(X, Y / \sim)$. Since $Y / \sim$ is completely regular, $H_{0}$ is relatively compact by Case 1 . This implies the conclusion by the openness of the map $q$. Q.E.D.

2. Sequential compactness. We give now some results on the equivalence between pseudocompactness and sequential compactness. Since the closure of a relatively countably compact subset is pseudocompact, the following theorem generalizes [4, Theorem 4] and [6, §18, Problem L]. Note that [4, Theorem 4] fails without some additional hypothesis on the range: a counterexample is given by taking constant maps into a countably compact space which is not sequentially compact. The assumptions on domains of the following results cannot longer be weakened since there are completely regular spaces which are countably compact but not sequentially compact, and every completely regular space is embeddable into a space $C_{p}\left(C_{p}(X)\right)$.

According to [2, 2 , Exercise 23], a topological space is called submetrizable iff its topology is finer than a metrizable topology. We shall say that a topological space is $\sigma$-pseudocompact iff it is the union of a sequence of pseudocompact subsets. Examples of submetrizable completely regular nonmetrizable spaces are: spaces of type $C_{p}(X, Y)$ with $X$ separable, $L F$-spaces.

2.1 THEOREM. If $X$ is a topological space with a dense $\sigma$-pseudocompact subset and $Y$ a submetrizable completely regular space, then every separable pseudocompact subset $H$ of $C_{p}(X, Y)$ is metrizable.

Proof. Let $\left(E_{n}\right)_{n=1}^{\infty}$ be a sequence of pseudocompact subsets of $X$ whose union is dense in $X$, and let $d$ be a metric whose topology is less fine than the topology of $Y$. By a well-known theorem of Kuratowski $(Y, d)$ is embeddable into a Banach space $B$, and we identify the maps $X \rightarrow Y$ with their natural corresponding maps $X \rightarrow B$. Let $Z$ be the topological vector subspace of $C_{p}(X, B)$ spanned by $H$. Since $H$ is a separable subspace of $C_{p}(X, Y), Z$ is separable: let $\left\{f_{n} \mid n \in Z^{+}\right\}$be dense in $Z$. The weak topology $\tau_{n}$ induced by $\left\{\left.f_{i}\right|_{E_{n}} \mid n \in Z^{+}\right\}$is pseudometrizable and less fine than the topology of $E_{n}$, hence bicompact by a well-known property of pseudocompactness. Then by [4, p. 175 , footnote], $\left.f\right|_{E_{n}}:\left(E_{n}, \tau_{n}\right) \rightarrow B$ is continuous for all $f \in Z$. Since every $\left(E_{n}, \tau_{n}\right)$ is separable and $\bigcup_{n=1}^{\infty} E_{n}$ dense in $X$, we conclude that there is a countable subset $E$ of $X$ such that

$$
\left.f\right|_{E}=\left.g\right|_{E} \Rightarrow f=g \quad(f, g \in Z)
$$

Consequently the origin of $Z$ is a $G_{\delta}$, being the intersection of its neighborhoods

$$
\{f \in Z|| f(x) \mid<1 / n\} \quad\left(x \in E ; n \in Z^{+}\right) .
$$


Then by a well-known property of neighborhoods based of vector topologies, $Z$ is submetrizable, so that the subspace $H$ of $Z$ is metrizable by [2, $\S 2$, Exercise 23-b)]. Again by [2, $\S 2$, Exercise 23-b)], $B$ induces on $H(x)$, $x \in X$, the same topology as $Y$, so that $H$ receives the same topologies from $C_{p}(X, B)$ and $C_{p}(X, Y)$. Q.E.D.

The following result may be added to a long list (see [5, Chapter vii]) of results which lead to conclude that products of separable spaces are in some way "almost separable".

2.2 COROLlaRY. If $X$ is an open subset of an arbitrary product $\prod_{\alpha \in A} X_{\alpha}$ of separable spaces and $Y$ a submetrizable completely regular space, then any separable pseudocompact subset of $C_{p}(X, Y)$ is metrizabie.

Proof. Let $H$ be a countable, relatively pseudocompact subset of $C_{p}(X, Y)$, and let $\bar{H}$ be its closure in $C_{p}(X, Y)$. Let us denote, for $B \subseteq A$, by $\pi_{B}$ the projection $\left(x_{\alpha}\right)_{\alpha \in A}-\leadsto \rightarrow\left(x_{\alpha}\right)_{\alpha \in B}$ restricted to $X$. By Gleason's theorem [5, vii. 23], to each $f \in H$ there corresponds a countable subset $C_{f} \subseteq A$ such that

$$
f=g_{f} \circ \pi_{C_{f}},
$$

where $g_{f}: \pi_{C_{f}}(X) \rightarrow Y$ is continuous. Define $C=\bigcup_{f \in H} C_{f}$. Since $H$ is countable, also $C$ is. The equality $f=g_{f} \circ \pi_{C_{f}}$ means that if two points $x, y \in X$ have the same coordinates for indices in $C_{f}$, then $f(x)=f(y)$. Consequently, if $x, y \in X$ have the same coordinates for indices in $C$, then $f(x)=f(y)$ for all $f \in H$, i.e.

$$
f=g_{f} \circ \pi_{C} \quad(f \in H),
$$

where $g_{f}: \pi_{C}(X) \rightarrow Y$ is continuous because $\pi_{C}$ is an open map (being the restriction on an open set of an open map). Choose $h \in \bar{H}$. There is a net $\left(f_{\beta}\right)_{\beta}$ in $H$ converging pointwise to $h$. Consider any $x, y \in X$ having the same coordinates in $C$. Then $f_{\beta}(x)=f_{k}(y)$ for all $\beta$. Hence

$$
h(x)=\lim _{\beta} f_{\beta}(x)=\lim _{\beta} f_{\beta}(y)=h(y)
$$

since limits are unique in a Hausdorff space. Therefore we may repeat a reasoning made above to obtain

$$
h=g_{h} \circ \pi_{C}
$$

with $g_{h}$ continuous. Then $h \rightarrow m \rightarrow g_{h}$ is a map $\varphi: \bar{H} \rightarrow C_{p}\left(\pi_{C}(X), Y\right)$. Obviously $\varphi$ is a homeomorphism into. By $2.1, \varphi(\bar{H})$ is metrizable since $\pi_{C}(X)$ is separable. Consequently $\bar{H}$ is metrizabie. Q.E.D.

2.3 REMARKS. (i) Concerning the sole equivalence between countable compactness and sequential compactness, 2.1 and 2.2 may be proved under the hypothesis "Each point of $Y$ is a $G_{\delta}$ ". This needs a result well known for compact spaces: A pseudocompact uniformizable space whose 
points are $G_{\delta}$-sets is 2 nd countable. (Its proof is somewhat more difficult than in case of compact spaces.)

(ii) The argument in the proof of 2.2 suggests a proof of the following result :

\section{If $X$ is a paralindelöfian space and $Y$ a metric space, then every pseudocompact equicontinuous subset $H$ of $C_{p}(X, Y)$ is metrizable,}

(the paralindelöfian spaces being defined in [1] and characterized topologically in [9]). We only briefly sketch the proof since this result is not entirely satisfactory by the assumption of equicontinuity of $H$. By [5, iii. 38], $H$ is equi-uniformly continuous with respect to the fine uniformity $\mu$ of $X$ and the metric of $Y$. Since $\mu$ has a base made of countable uniform covers, $\mu X$ is embeddable into a product of separable metric spaces. Now we repeat the argument in the proof of [10, Theorem] to factor each $f \in H$ into $f=g_{f} \circ \pi_{C}$ with $\pi_{C}$ a fixed countable projection and $\left\{g_{f} \mid f \in H\right\}$ equi-uniformly continuous. Then we conclude as in the case of 2.2.

\section{REFERENCES}

1. G. Aquaro, Una generalizzazione degli spazii di Lindelöf, Ann. Scuola Norm. Sup. Pisa (3) 16 (1962), 195-206. MR 26 \#5535.

2. N. Bourbaki, Éléments de mathématique. I: Les structures fondamentales de l'analyse. Fasc. VIII. Livre III: Topologique générale. Chap. 9: Utilisation des nombres réels en topologie générale, 2ième éd., Actualités Sci. Indust., no. 1045, Hermann, Paris, 1958; English transl., Hermann, Paris; Addison-Wesley, Reading, Mass., 1966. MR 30 \#3439; MR 34 \#5044b.

3. H. H. Corson, Normality in subsets of product spaces, Amer. J. Math. 81 (1959), 785-796. MR 21 \#5947.

4. A. Grothendieck, Critères de compacité dans les espaces fonctionnels généraux, Amer. J. Math. 74 (1952), 168-186. MR 13, 857.

5. J. R. Isbell, Uniform spaces, Math. Surveys, no. 12, Amer. Math. Soc., Providence, R.I., 1964. MR 30 \#561.

6. J. L. Kelley and I. Namioka, Linear topological spaces, The University Series in Higher Math., Van Nostrand, Princeton, N.J., 1963. MR 29 \#3851.

7. S. Mazur, On continuous mappings on Cartesian products, Fund. Math. 39 (1952), 229-238. MR 14, 1107.

8. V. Pták, On a theorem of W. F. Eberlein, Studia Math. 14 (1954), 276-284. MR 16, 595.

9. G. Vidossich, Two remarks on A. Gleason's factorization theorem, Bull. Amer. Math. Soc. 76 (1970), 370-371. MR 41 \#1021.

10. - Topological characterization of pseudo-א-compact spaces, Proc. Amer. Math. Soc. 27 (1971), 195-198. MR 42 \#2434.

Department of Mathematics, University of Pisa, Pisa, Italy

Current address: Scuola Normale Superiore, 56100 Pisa, Italy 